\title{
Breathtaking advances in stem cell biology
}

\author{
John A Kessler
}

Substantial publicity was generated by the recent report that it is possible to clone primate embryos by somatic cell nuclear transfer and to derive embryonic stem cells (ESCs) from these embryos (Byrne JA et al. [2007] Nature 450: 497-502). Even greater public fanfare greeted two almost concurrent reports that human skin fibroblasts can be directly reprogrammed into pluripotent cells that closely resemble ESCs (Takahashi et al. [2007] Cell 131: 861-872; Yu J et al. [2007] Science 318: 1917-1920). These reports raise two important questions. First, are these findings real? Second, will their impact on the fields of stem cell biology and medicine be as profound as the media imply? Happily, the answer to both questions is yes!

The first question about whether the findings are real is one that should greet all reports of major scientific advances. The previous fraudulent claims by Hwang and others of similar findings with human cells have, however, created an additional layer of skepticism, and Nature took the unusual step of soliciting independent verification of the Byrne et al. paper during the peer review process (Cram DS et al. [2007] Nature 450: E12-E14). Corroboration was relatively straightforward, did not delay publication, and raised confidence in the findings, so the overall outcome of Nature verifying the findings was positive in this case. Some discoveries will, however, be more difficult to verify, and the final responsibility for corroborating scientific advances must ultimately lie with the scientific community.

Most stem cell biologists believed that cloning of primate embryos required technical but not conceptual breakthroughs, and were not unduly surprised by the findings of Byrne and colleagues. Most found it breathtaking, however, when Takahashi and Yamanaka reported that adult mouse fibroblasts could be reprogrammed into pluripotent cells (induced pluripotent stem cells, or iPS cells) by viral overexpression of only four genes (Takahashi K
.... it should be

possible to

use a patient's

own skin cells

to create

pluripotent

cells for use

in tissue

regeneration

or cellular gene

therapy

JA Kessler is an Advisory Board member of Nature Clinical Practice

Neurology.

\section{Competing interests}

The author declared no competing interests.

www.nature.com/clinicalpractice doi:10.1038/ncpneuro0751 and Yamanaka S [2006] Cell 126: 663-676). In this case, verification occurred through the usual scientific process - several other laboratories corroborated the findings within a year. The more recent and highly publicized reports cited above demonstrated that the same can be done with human fibroblasts.

The potential implications for medicine are enormous. These studies demonstrate that it should be possible to use a patient's own skin cells to create pluripotent cells for use in tissue regeneration or cellular gene therapy. Such cells would contain the patient's own DNA, so they could be transplanted into damaged organs without the need for immunosuppression and without any inherent genetic mismatch with surrounding cells. Nevertheless, the road to therapeutic applications will be neither easy nor rapid. Ethical issues surrounding somatic cell nuclear transfer and the need to harvest human oocytes handicap this approach to such an extent that its clinical utility is uncertain. It also remains unclear whether the potentials of iPS cells and ESCs are identical, and there is substantial concern about the risk of cancer after transplantation of iPS cells. Before these cells can be used clinically, techniques must be developed for controlling the insertion sites of viral constructs into host cell DNA, regulating the expression of the viral constructs, and perhaps excising them before clinical use. It will also be necessary to learn how to differentiate cells into appropriate phenotypes that survive and integrate into host tissues.

Despite many remaining technical obstacles, the recent discoveries demonstrate that it should be possible to develop new therapies with use of patient-specific pluripotent cells. Neurologists can begin to think about personalized cell replacement therapies for diseases such as Parkinson's disease and Huntington's disease, and personalized cellular gene therapy for lysosomal storage diseases and other inherited disorders of the nervous system. 